\title{
Le sacrifice et son étude du point de vue néoplatonicien
}

Sacrifice and its study from a neo-Platonic viewpoint

\section{Albert de Surgy}

\section{(2) OpenEdition}

\section{Journals}

Édition électronique

URL : http://journals.openedition.org/span/347

DOI : 10.4000/span.347

ISSN : 2268-1558

Éditeur

École pratique des hautes études. Sciences humaines

\section{Édition imprimée}

Date de publication : 1 septembre 1976

Pagination : 205-230

ISSN : 0294-7080

\section{Référence électronique}

Albert de Surgy, «Le sacrifice et son étude du point de vue néoplatonicien », Systèmes de pensée en

Afrique noire [En ligne], 2 | 1976, mis en ligne le 02 juillet 2013, consulté le 19 avril 2019. URL : http:// journals.openedition.org/span/347 ; DOI : 10.4000/span.347 
L E S A C R I F I C E E T S O N É T U D E

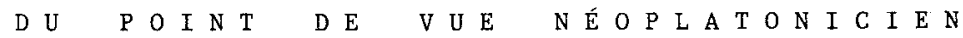

par

Albert de Surgy

Contrairement à certaines idées reçues, la théorie néoplatonicienne du sacrifice, êlaborée au contact des cultes égyptiens et proche-orientaux, correspond étroitement à la mentalité et aux pratiques religieuses des Noirs d'Afrique (occidentale).

Comme la philosophie néoplatonicienne est avant tout une réflexion sur $l^{\prime}$ agencement et 1 'interaction des principes directeurs de notre univers, et brille d'autre part par le caractère rationnel, ëminemment dialectique, de ses développements, nous devons nous attendre à y trouver des séries de concepts et de suggestions particulièrement utiles à toute approche scientifique des activités religieuses.

Une étude du sacrifice en Afrique noire ne saurait en ignorer les principales ressources sans se priver volontairement d'une de ses meilleures chances de succès.

L'étude des conceptions religieuses et philosophiques des anciens Grecs présente pour l'ethnologue africaniste un double intérêt.

- En effet, des pratiques divinatoires et religieuses analogues à celles que nous pouvons éncore observer en Afrique étaient courantes dans le monde grec. Nous trouvons chez les auteurs anciens des éléments de comparaison qui nous permettent de mieux connâ̂tre les variétés de pratiques en cause et de juger des influences qui se sont éventuellement exercẻes d'une région à l'autre (1).

(1) Ainsi, les cultes de possession en Afrique occidentale ont d'étroites ressemblances et d'étroits rapports historiques avec les orgies dionysiaques et le corybantisme en général. 
- Mais surtout les philosophes grecs, en particulier ceux qui se rêclamaient de Pythagore et de Platon, se sont penchés sur ces pratiques et ont tentê d'en fournir une explication rationnelle. Dans le cadre de leurs théories, ils ont forgé un vaste ensemble de concepts appropriés à leur objet, c'est-à-dire permettant de traiter des faits envisagés sans les rêduire à autre chose que ce qu'ils sont, devent

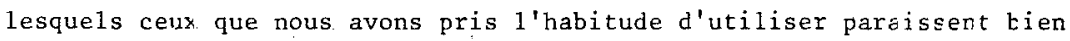
pâles.

Il serait dommage de ne pas tenter de tirer parti de tels travaux, bien puéril de croire que tout commence avec Lévy-Bruhl ou avec Mauss et qu'auparavant ne régnait que vide et déraison.

Prenons deux exemples :

1) Plusieurs d'entre nous ont rencontré des notions d'oeuf du monde contenant deux principes opposés associés l'un à l'autre. Que recouvre exactement cette notion? Quelles difficultés logiques résoutelle ou soulève-t-elle ? Quelles peuvent en être les conséquences sur le développement d'une cosmogonie ? Il ne fait aucun doute que les investigations de quiconque en ce domaine seront facilitées, car il saura dès lors formuler des hypothèses plus prêcises, s'il a déjà pris connaissance des aventures de I'oeuf orphique. Cet oeuf fut pondu dans l'Ether par Chronos et il en sortit Phanès, le premier né des dieux. Ce dieu était double; on dit qu'il avait deux paires d'yeux (comme Janus-Aǐon, immortel) et qu'il était à la fois "Femelle et Père" (Proclus). Plus tard, Zeus avale Phanès et $i 1$ en résulte un nouveau monde ou une récréation du monde sous 1 'empire de Zeus.

2) Nous sommes largement confrontés, les uns et les autres, à des cultes de jumeaux; mais il y a jumeaux et jumeaux; car la notion de deux principes jumelés se reflète de diverses manières à divers niveaux du Tout. Il ne fait aueun doute que nos moyens conceptuels d'investigation seront augmentés si nous nous sommes penchés sur les distinctions à établir entre :

- Les Cabires assimilés à Hadès et à Hermès,

- Les Dioscures : Castor et Pollux,

- Les deux Castores dolichéniens,

- Cautopatès et Cautès, les dadophores du culte de Mithra,

- Les couples d'Attis;

- Héraklès et Iphik1ès, 
Le sacrifice du point de vue néoplatonicien

- Eros et Antéros,

- Phosphoros et Hespéros etc...

Enfin, n'avons-nous pas tendance à considérer comme deux principes jumeaux les composants de toute dyade (ne dit-on pas que le Ciel est le jumeau de la Terre) ?

Mais les anciens ne nous ont pas proposé uniquement divers concepts ; ils nous proposé aussi une attitude et une méthode. A leur avis, on ne peut bien connaître un phénomène que si l'on a eu contact avec lui et l'on ne peut connaitre un être sans devenir semblable à lui, autrement dit, il n'y a pas de connaissance sans expérience, expérience qui modifie quelque chose en nous.

Vous êtes convaincus, j'imagine, que pour traiter convenablement d'une population, il faut avoir eu contact avec cette population. Dans le cas contraire, l'esprit "déraille", la pensée tourne à vide à l'intérieur d'un système ou d'une doctrine qui en vient à nier, puis à déttruire,toute autre forme d'existence, et qui en vient à fabriquer une "science" qui appauvrit l'être au lieu de le-servir.

Or, certes, nous avons les uns et les autres un contact épisodique avec le terrain. Mais à ceux qui entendent traiter de religion, de divination, de possession ou de magie, il manque presque toujours un contact avec les puissances en jeu dans la religion, la divination, la possession ou la magie.

Comment peut-on traiter sérieusement de ces sujets sans croire qu'ils recouvrent une réalité originale et sans avoir, d'une manière ou d'une autre, 1'expérience personnelle de cette réalité ? Ou nous croyons que les phénomènes dits. "religieux" sont autre chose qu'un ramassis d'épiphénomènes incohérents, ont une nature spécifique et sont soumis à certaines lois, ou nous devons, en tant que scientifiques, nous désintérésser immédiatement de ces phénomènes et en confier 1 'examen à d'autres disciplines (psychologie, sociologie, économie...) ou aux amateurs d'extravagances !

C'est un sportif que $I^{\prime}$ 'on enverrait étudier la pratique du sport dans une autre partie du monde, un musicien étudier la musique, un technicien étudier la technique etc... De même convient-il d'envoyer étudier la religion et la divination en tant que telles des individus éduqués en matière de thêologie, de démonologie et de pratiques initiatiques. 
N'oublions pas que les philosophes dont nous allons parler : Platon, Jamblique etc... étaient qualifiés de "divins" en raison de leur fréquentation des dieux. N'oublions pas que Proclus, qui est le premier à avoir êlaboré une "théologie scientifique" de la nature d'une théodicée, était un faiseur de pluie et faisait apparaître des dieux. Il savait donc de quoi il parlait. Il disait de la théurgie (In Plat. Théol., I, 25 ; Budé p. 113) : qu'elle "est meilleure que toute sagesse et toute science humaine puisqu'elle concentre en elle les avantages de la divination, les forces purificatrices de l'accomplissement des rites et tous les effets sans exception de l'inspiration qui rend possêdé du divin".

Voilà un logicien de premier ordre dont son biographe Marinus nous parle de ses "longues vigiles d'adoration la nuit" et de "ses triples prosternations devant le soleil : à son lever, à son midi et à son coucher" (Vita Procli, 22), un écrivain qui renonça à un commentaire sur les écrits orphiques à la suite d'une interdiction de son maître défunt Syrianus qui lui apparut en songe, un thaumaturge dont la prière sut guérir la fille unique d'Archiadas et de Ploutarchè (Vita Procii, 29).

L'idéal exigerait que la science religieuse soit faite avec des hommes de cette trempe ayant l'expérience personnelle du sacré, que l'étude du sacrifice soit accomplie par des sacrifiants sachant introduire le sacrifice dans leur "laboratoire". P1us modestement, contentons-nous de recommander une formation aux disciplines qui, selon Proclus (In Plat. Théol., I, 2 ; Budé pp. 10-11) doivent servir de propédeutique à la théologie scientifique dont la perspective rejoint celle de la science des religions : la pratique des vertus morales; 1 'exercice logique et dialectique; l'étude des sciences physiques ou naturelles ; l'étude des mathématiques (2). Et souhaitons que l'investigation ethnographique soit menée en considérant les informateurs comme des sujets au même titre que nous, ayant aussi droit de regard sur nous, pouvant devenir pour nous une source d'enrichissement personnel.

(2) Même doctrine chez Ammonius, Simplicius, Philipon, Elias et Olympiodore. 
Incontestablement, la réflexion théorique rationnelle d'inspiration orphique, pythagoricienne et platonicienne, susceptible de nous être utile dans nos recherches sur le sacrifice en Afrique noire, trouva son plein épanouissement dans le mouvement néoplatonicien qui se poursuivit au cours des IIIe, IVe et Ve siècles après Jêsus Christ. Il est clair que la philosophie néoplatonicienne constitue à elle seule tout un monde, que ses plus éminents dëfenseurs se sont maintes fois porté la contradiction et ont évolué, qu'il conviendrait de rendre compte de cette richesse en les éclairant les uns par les autres (3). Dans le cadre limité de cet exposé, je ne saurais entrer dans le détail de tous ses aspects. Je ne saurais non plus en préciser les principales organisations conceptuelles, en particulier les principes de classification des dieux par ordres et par genres. Une telle classification existe et se trouve à la disposition de tous ceux qui ont l'ambition de penser les réalités religieuses à l'aide de termes nombreux et précis.

Je me limiterai ci-dessous à l'exposé de quelques grandes idées communes qui feront sentir, je l'espère, la profonde affinité de cette philosophie avec la mentalité et les réalités africaines. C'est à mon sens la seule tentative d'analyse rationnelle des phénomènes d'ordre religieux qui ait été faite dans une perspective identique à celle de la plupart des populations que nous étudions.

Selon donc les philosophes nêoplatoniciens :

a) Les dieux n'ont pas besoin des sacrifices que leur offrent les hommes et, a fortiori, des nourritures que ceux-ci déposent à leur intention. Quant au Dieu suprême, il n'a même pas besoin de culte.

"La divinité elle-même ne manque de rien ; si nous lui rendons les honneurs; c'est en vue de notre avantage. La providence des dieux s'étend de tous côtés, il n'est besoin pour la recevoir que d'une accommodation. Or, 1 'on s'accommode par imitation et ressemblance... De tout cela ensemble (c'est à dire des divers aspects dó culte), il ne vient aux dieux nul profit - quelle sorte de profit pourrait-il y avoir pour un dieu ? - mais c'est nous qui en tirons bénéfice de nous unir aux dieux." (Sallustius - XV)

(3) On pourra consulter, de J. Trouillard, Le néoplatonisme de Plotin à Damascios, contribution à l'Histoire de la Phizosophie I de I'Encyclopédie de la Pléiade, Paris, Gallimard, 1969. 
"Les sacrifices doivent être différents suivant les diverses puissances auxquelles ils sont offerts. On ne doit rien présenter au Dieu suprême, ainsi que $1^{\prime}$ a dit un sage, car ce qui est matériel est indigne d'un être qui est dégagé de la matière. C'est pourquoi il est inutile de s'adresser à lui, ou en lui parlant, ou même intérieurement... Il faut donc qu'en nous unissant à lui et en lui ressemblant, nous devenions une sainte hostie qui lui serve de louange... La perfection du sacrifice consiste à dêgager son âme des passions et à se livrer à la contemplation de la divinité."

"Quant aux dieux qui ont pour principe ce premier être... sacrifier à chacun les prémices des biens qu'ils nous donnent... offronsleur de bonnes pensées et remercions-les de ce qu'ils nous ont donné le pouvoir de les contempler, de ce que cette contemplation est la vraie nourriture de 1 'âme." (Porphyre, De abstinentia, II, XXXIV)

Pour Porphyre, le culte de tels dieux exclut tout sacrifice sanglant, car ils ne sauraient être intéréssés par des offrandes aussi vulgairement matérielles :

"Les meilleurs prémices que 1 'on puisse offrir aux dieux, ce sont un esprit pur et une âme dégagée des passions." (Tbid., II, LXI)

Dans son acception la plus haute, le sacrifice est l'occasion pour l'âme de trouver sa nourriture dans la contemplation divine, puis même dans le contact pris avec Dieu, mais ses buts et ses fruits sont d'ordre moral : imiter Dieu.

"Faisons tout ce qui dépendra de nous pour tâcher de nous rendre semblable à Dieu et aux bons génies ; et nous y parviendrons si, en nous guêrissant des passions, nous tournons toutes nos pensées vers les vrais êtres afin qu'ils nous servent continuellemnt de modèle et que nous évitions de ressembler aux méchants hommes ou aux mauvais génies." (Ibid., II, XLIII)

Dieu se définit avant tout comme le Bien. Prendre Dieu pour modèle, devenir semblable à Dieu, ce sera, avec lui et comme lui, rayonner autour de nous une bonté inépuisable, une bonté, par conséquent, qui ne saurait prendre'source et se constituer au niveau des réalitês corruptibles du monde matériel.

De fait, en Afrique, le culte du Dieu suprême est si effacé qu'il en devient pratiquement inexistant,ce dont de nombreux missionnaires n'ont pas manqué de s'indigner. Or ce n'est pas là négliger Dieu, de qui tout procède, mais le placer à son rang.

Dans sa thèse sur La notion du Dieu créateur dans les croyances traditionnelies (documents C.E.R.K., 1968, fasc. I), Seth Nomeyo, après avoir affirmé (p. 19) que "Mawu possède la plénitude de toutes choses et n'a pas besoin de 1'homme pour être ce qu'il est", nous 
Le sacrifice du point de vue néoplatonicien

montre que le culte de Mawu (1e Dieu suprême des Evhé) se définit avant tout par le respect des valeurs morales : "Si l'on veut savoir la place qu'occupe Mawu dans la vie des Evhé, il faut chercher cette place, non pas dans leurs pratiques religieuses, mais dans leur vie morale." (p. 32)

De plus, en pays evhê, le culte des dieux les plus importants non seulement se déroule dans une athmosphère de profond recueillement qui contraste du tout au tout avec la violence qui accompagne fréquemment les cêrémonies du culte des vodu, mais encore exclut tout sacrifice sanglant. C'est ainsi qu'un simple oeuf pourra parfois être abandonné dans une calebasse, sur pied fourchu, pour Mawu ; c'est ainsi que dans la cour des prêtres de Nyigbla, divinité nationale des Evhé du littoral, il est interdit de verser le sang; c'est ainsi que les plus importantes divinitês (tro) de la ville d'Anloga (l'un des principaux centres religieux evhé) se font offrir essentiellement de la bière de mil et des boulettes crues de farine de mil imbibées d'eau et de miel ; 1'une d'elles se voit offrir une chèvre abandonnée vivante à son intention en pleine campagne.

b) Les sacrifices ne profitant pas aux dieux, la question qui se pose alors est de savoir s'ils profitent aux démons.

Pour Porphyre : "Il faut que celui qui s'applique à la piété ne sacrifie aux dieux rien d'animé, mais seulement aux démons, soit bons soit mauvais." (De abstinentia, II, XXXVI)

"C'est une chose avérée chez les théologiens que 1 'on offrait des sacrifices ensanglantés non aux dieux, mais aux démons." (Ib́id, II, LVIII)

Il nous explique, en ce qui concerne les mauvais démons, qu'"ils prennerit plaisir aux sacrifices ensanglantés. Ce qu'il y a en eux de corporel s'en engraisse, car ils vivent de vapeurs et d'exhalaisons et se fortifient par les fumées des sangs et des chairs." (Ibid., II, XLII)

Mais, pour Jamblique, cette idée que les démons pourraient avoir matériellement besoin d'être nourris par les sacrifices est absurde, car :

"De se laisser séduire par l'exhalaison des vapeurs, déjà un homme sensé et sans passions ne pourrait s'y résoudre, encore moins un des êtres supérieurs." (De mystépiis, V, 4 / 205)

"Si les démons sont entourés d'un corps, que certains croient nourri par les sacrifices, ce corps est invariable, impassible, lumineux, sans besoins, en sorte que rien n'émane de lui et qu'il se passe 
d'émanations de l'extérieur. Et si même par hasard on posait cela, puisque le cosmos et l'air qu'il renferme reçoivent sans arrêt 1 'exhalaison de ce qui se fait sur terre et se répandent de toutes parts à 1 'égal de pareils effluves, quel besoin les démons ont-ils des sacrifices ?...Il serait inconcevable que le Démiurge eût à tous les animaux terrestres et marins servi copieusement et toute prête leur nourriture et qu'il en eût privé les êtres supérieurs à nous...

"Pourquoi donc ceux qui s'expriment de la sorte ne vont-ils pas jusqu'à renverser toute la hiérarchie... car s'ils font de nous les nourriciers et les pourvoyeurs des démons, nous serons avant eux dans $l^{\prime}$ échelle des causes... s'il est impossible que nous soyons les causes premières des démons, il n'est pas plus vrai qu'ils nous doivent aussi leur nourriture." (Ibid., v, 10/212-213)

L'opinion selon laquelle les démons se nourrissent d'exhalaisons et d'odeurs est très largement partagée (par exemple, Plutarque, De facie..., p. 943 e). Si tel était le cas, il y aurait par là un moyen matériel de les atteindre. L'objection de Jamblique n'en garde pas moins tout son poids : que pourraient ajouter les sacrifices à la somme des exhalaisons terrestres dont ils ont jouissance à profusion ?

Cependant, à supposer que les démons ne trouvent pas matériellement profit aux sacrifices, peut-être y trouvent-ils profit sur le p1an émotionne1, de la même manière qu'un homme dénué de besoins s'émeut du plus simple des cadeaux. Pour Plutarque, Porphyre et Apulêe notamment, les démons sont des êtres sensibles et sujets aux passions, car constitués d'un principe ayant même nature que 1 'âme, uni à un corps, ou véhicule de souffle, dont l'habitat est l'air.

"Ils (les bons démons) sont produits du plus pur et du plus parfait élément de l'air... En un mot, les démons, les génies ou les esprits familiers ont la raison en partage et sont soumis au joug des passions. Leur corps est de nature aérienne et ne périra jamais." (Apulée, De deo Socratis, pp. 53 et 61)

"Leur esprit (des mauvais démons), en ce qu'il est corpore1, est sujet aux passions et est corruptible, et quoiqu'il soit joint à leur âme pour être uni avec elle un très long temps, il n'est pas éternel, car il y a apparence qu'il en sort des écoulements et qu'il se nourrit." (Porphyre, De abstinentia, II, XXXIX)

"Typhon, Osiris et Isis... grands démons... mais ils n'ont pas eu la divinité pure et simple, ainsi ont étê un suppôt composé de nature corporelle et spirituelle, capable de volupté, de douleur et des autres passions et affections qui accompagnent ces mutations." (Plutarque, De Iside et Osimide, 25 ss., p. 360 d.)

"Il existe certains êtres, situés dans une zone limitrophe pour ainsi dire entre les dieux et les hommes, qui sont soumis aux passions des mortels et aux vicissitudes de la fatalité, et qu'il est juste... 
que nous considérions comme des démons... (selon Xénocrate) la nature des démons participe à la fois à la passibilité des mortels et à la puissance de la divinité." (Plutarque, De defectu Or., 416, C et D)

Philon, au contraire, parlant des démons, nous dira que :

"Ces âmes ne sont pas des mélanges de raison et d'irrationnel, mais l'irrationnel en est retranché ; elles sont intelligibles dans toutes leurs parties." (De Monarchia, II, I, p. 222)

I1 les définit comme des "essences pneumatiques" (Quaest. in Gen., I, 92, Harris 18), des "esprits sans corps" (Ibid., III, II et IV, 188).

Jamblique estime, pareillement, que les démons sont tout aussi impassibles que les dieux :

"Puis donc que, dans le cas du dernier des genres supérieurs, celui de l'âme, nous avons démontré impossible la participation au pâtir, pourquoi faut-il l'attacher aux démons et aux héros ? ... par conséquent, impassibles sont aussi les dẻmons et tous ceux des genres supérieurs qui les escortent." (De mystériis, I, 10 / 36)

"Ils font partie des genres supérieurs dont les êtres sont séparés, ayant leur substance par eux-mêmes, non du fait d'autrui ou en d'autres." (Ibia., I, 4 / 13)

La suite du texte montre qu'il limite la notion de démon à l'âme, ou tout au moins à la partie essentielle des démons qui n'exclut pas une certaine corporéité :

"Ce n'est ni de la matière ni des éléments ni d'aucun autre des corps de nous connus qu'est fait le véhicule quasi-corporel au service des démons. Quelle sorte de complément une essence pourraitelle apporter à une autre essence ?" (Ibid., V, $12 / 215$ )

Proclus réexprimera la même idée avec encore plus de vigueur dans ses Eléments de théologie:

Théorème 196 : "Toute âme participable jouit d'un corps premier et perpétuel qui n'est pas soumis dans sa substance à la naissance ni à la corruption."

Théorème 208 : "Le véhicule de toute âme particulière est immatériel, indivisible en sa substance et impassible."

Ces affirmations le contraignent à adjoindre à l'âme deux autres vêhicules : un vêhicule intermêdiaire qui rend l'âme citoyenne du devenir et un dernier vêhicule terrestre, à savoir le corps (In Timaeum, III, 297, I-3 et 285, 298, 299).

En effet (Théorème 209), "le véhicule de l'âme particulière descend en s'ajoutant des tuniques matérielles." 
Nous yoyons dès lors que les divergences entre les auteurs viennent de ce que les uns considèrent les démons (âmes démoniques) indépendamment de leur vêhicule matériel subtil (corps subtil ou corps de souffle), tandis que les autres les considèrent, descendus parmi nous dans 1 'air, en association avec ce vêhicule.

Semblablement, Jamblique se trouve contraint d'associer aux démons proprement dits "la puissance cosmique ou terrestre des démons ou des dieux" (De mystériis, V, 9 / 210), nêcessaire pour justifier toutes les composantes matérielles des sacrifices adressês non seulement aux démons mais aussi aux dieux cosmiques :

"Les dieux matériels, donc, ont une certaine communauté avec la matière en ce qu'ils trônent sur elle; ... si $l^{\prime}$ 'on veut honorer de tels dieux par la théurgie, c'est selon leur nature et l'autorité qui est leur lot qu'il faut leur rendre un culte, matériel comme eux.. - (les composantes matérielles du sacrifice) conviennent aux dieux qui président à la matière préexistante; non aux dieux par eux-mêmes, mais en raison de la matière sur laquelle ils règnent. Car même s'ils en sont séparés le plus possible, néanmoins ils lui sont présents ; même s'ils l'enveloppent en vertu d'un pouvoir immatériel, ils sont avec elle. Les administrés ne sont pas étrangers aux administrateurs ni les êtres ordonnés aux ordonnateurs, et ce qui sert n'est pas, en tant qu'instrument, sans accord avec $l^{\prime}$ 'usager. C'est pourquoi offrir de la matière dans les sacrifices aux dieux immatériels est chose hors de propos, mais cela convient parfaitement aux dieux matériels." (Ibid., v, $14 / 218$ )

Si les sacrifices matêriels conviennent aux dieux "matériels", a fortiori conviendront-ils aux démons qui constituent un genre inférieur à celui des dieux.

Les démons, entièrement incorporels, ne peuvent pas entrer directement en communication avec des êtres corporels. Il leur faut pour cela utiliser un véhicule intermédiaire ayant contact avec le plan matériel. Ce véhicule leur est fourni par l'âme animale - soit par le psychisme animal de 1 'homme dans le cas d'une communication par "possession", soit par une âme animale désincarnée par suite d'un sacrifice sanglant.

Voici donc justifiés, dans une perspective spirituelle des plus rigoristes, mais certes à des fins dont l'auteur conseille de se détourner pour en adopter de plus hautes, à la fois les cultes de possession et les immolations d'animaux :

"Au démon qui est complètement incorporel et ne reçoit de nulle par la corruption, le corps ne communique pas de souillure." ( $I$ bid., 214 
VI, 2 / 242)

"Qu'aux démons... soit attribué ce contact avec les instruments divinatoires; ou, $s i$ on le préfère, qu'on leur impartisse un siège qui leur permette de fréquenter les hommes et de s'en servir ; ce siège là aussi, il faut le concevoir pur de tout corps, car il n'y a pas de communication entre le pur et son contraire; et c'est vraisemblablement par l'âme des animaux qu'il se rattache aux hommes. Cette âme, en effet, a quelqu'affinitê avec l'homme par l'homogénéĩté de leur principe vital ; avec le démon aussi, parce que, délivrée du corps, elle se trouve en quelque manière séparée ; étant intermédiaire entre les uns et les autres, elle sert celui qui a autorité sur elle et annonce à ceux que le corps retient encore ce que lui enjoint le démon qui la gourverne; elle donne à ces deux espèces un lien commun qui les unit..."

"Or un mode d'oracle tel que celui-ci est tout à fait distant du mode divin et vrai, capable qu'il est de prédire l'avenir en des matières mesquines et ëphẻmères, celles que comporte la nature divisée et qui concernent déjà le créé ; il produit des mouvements autonomes en qui peut les recevoir et provoque de mille manières des passions en qui a disposition et aptitude à sympathiser ; mais ce n'est pas par une passion que pourrait jamais se produire la prescience parfaite." (Ibid., VI, 3 et $4 / 243-244$ )

Les dieux ne tirant aucun avantage des sacrifices, nous comprendrons le repas sacrificiel comme le symbole de l'entretien avec les dieux consécutif à la descente des dieux suscitée par le sacrifice lui-même. Le dieu n'a que faire de la charité humaine, mais il vient se communiquer aux hommes, par pur effet de sa bonté, en participant finalement à leur assemblée.

Dans la mesure où nous associons au dieu la puissance cosmique ou terrestre qui lui a été attribuée, ou dans la mesure où nous associons de plus au démon son vêhicule de souffle, nous admettons qu'il pactise avec les hommes en échangeant avec eux, rituellement, des biens dont il n'a matériellement aucun besoin, mais qui font tout simplement partie de son apanage. Cet échange ne tient lieu pour lui de nêcessité que s'il se trouve passionnément intéressé à participer aux affaires des hommes. Tel est le cas des esprits humains désincarnés qui, après la mort, ne sont pas parvenus à se détacher pleinement du monde où ils ont vécu (ces "génies ayant vécu dans un corps mortel", ces Lémures, Larves ou Mânes dont nous parle Apulée). Tel est aussi le cas des principes actifs analogues appelés "mauvais démons", mais qui ne sont mauvais que relativement à une perspective de libération de l'âme humaine et relativement à qui en subit l'influence (Jamblique, Ibid., I, 18). 
Par opposition à ceux qui agissent en sens contraire (4), ces démons, en effet, encouragent les manifestations sur les plans inférieurs de l'être, à proximité du chaos vers lequel est orientée l'action de Nature (Physis), de sorte que leur rôle est de provoquer partout épaississement, obscurcissement, effervescence, confusion, terreurs, drames, transformations, en même temps que richesses, surabondance, excès de toutes sortes :

Selon Jamblique, "les dieux sont affranchis des puissances inclinées vers le devenir, mais les démons n'en sont pas complètement purs." (Ibid., I, 20/64)

"Il faut réserver aux démons des puissances fécondantes, qui président à la Nature et au lien des âmes avec les corps", ... et les activités des démons "sont davantage cosmiques" (Ibid., II, 1, 2 / 6768).

"Les démons tirent vers la nature...

"Aux démons se mêlent des vapeurs cosmiques et elles se meuvent sans repos au gré du mouvement du monde." (Ibid., II, 5 / 79-80)

La venue "des démons alourdit le corps, le châtie par des maladies, entraîne l'âme vers la nature, n'éloigne pas des corps et de la sensation qui leur est apparentée, retient dans les régions d'ici-bas ceux qui s'empressent vers le feu, ne libère pas des liens de la fatalité" (Ibid., II, 6/82).

Selon Porphyre: "Toutes les âmes qui ont pour principe l'âme de l'univers gouvernent les grands pays qui sont situés sous la lune. Leur administration est conforme à la raison. Il faut être persuadés que ce sont de bons démons... Mais toutes les âmes qui, au lieu de dominer l'esprit qui leur est uni, s'en laissent gouverner jusqu'à être transportées par la colère et par les passions, sont avec raison appelées démons malfaisants." (De abstinentia, XXXVIII)

"Ils inspirent un amour violent des richesses, des honneurs, des plaisirs, de la vaine gloire, source des divisions et des guerres." (Ibid., XL)

"Nous n'examinons point si c'est une nécessité aux villes de les apaiser. On y regarde les richesses et les choses extërieures, corporelles, comme de vrais biens, et le contraire comme des maux. On y est fort peu occupé du soin de l'âme. Pour nous, autant qu'il sera possible, n'ayons pas besoin des faveurs de ces génies..." (ibid., XLIII).

"Moins nous aurons de soin de nous dégager de nos passions, plus nous dépendrons des mauvaises puissances et plus il sera nécessaire de

(4) De même que le Démiurge, ou Soleil Intelligible, se différencie en dieux célestes recevant la direction des astres ou corps célestes vers lesquels se tournent une partie des démons, de même la Lune intelligible se différencie en une multitude de puissances féminines commandant par séduction à une multitude de "démons terrestres". 
leur sacrifier pour les apaiser." (Ibid., XLIII)

Dans ces conditions, le plaisir que prendraient les (mauvais) démons aux sacrifices sanglants et aux orgies viendrait de ce que, par essence, ils prennent plaisir à tout ce qui dégrade ordre et beauté. I1 y aurait soulagement et jouissance, de caractère orgasmique, chez un dêmon (d'où leur union de caractère sexuel avec ceux qui les adorent) dès que se produirait à leur instigation une chute de principe vital, depuis le plan de la sérénité parfaite jusqu'au plan de la génération et de la corruption où prolifèrent les biens terrestres et tous les maux qui en sont l'inévitable contrepartie.

Si l'abstinence de tels sacrifices parâ̂t être de rigueur pour Porphyre dans une perspective étroitement purificatrice, elle ne semble pas prônée par Jamblique pour qui, d'un point de vue théurgique plus puissant et transfigurant, l'épanouissement corporel, dénué de tout attachement, ne semble pas faire obstacle, bien au contraire à la communication et encore moins à la communication suprême avec les dieux.

c) Livré à ses propres forces, 1'homme est absolument incapable de se hausser jusqu'aux dieux ou d'amener les dieux à se manifester jusqu'à lui, de façon à être inondê de grâces divines. Si donc le sacrifia porte fruit, il faut en rechercher uniquement la raison auprès des dieux eux-mêmes.

Ceci, Jamblique nous 1 'explique admirablement :

"Quel est le rituel, en effet, quel est le culte célébré selon les lois hiératiques qui s'accomplit par la passion ou produit une satisfaction des passions ? Le culte n'a-t-il pas été codifié au début selon les dêcrets des dieux et intellectuellement ? Et il imite 1 'ordre des dieux, 1 'intelligible et celui du ciel. Et il comporte des mesures éternelles de ce qui est et des signes admirables, vu qu'ils ont été envoyés ici-bas par le démiurge et père de tous les êtres. Grâce à eux, l'indicible s'exprime en symboles mystérieux..." (De mystériis, I, 21-65).

"C'est I'accomplissement religieux des actions ineffables dont les effets dépassent toute intellection, ainsi que le pouvoir des symboles muets, entendus des dieux seuls, qui opèrent l'action théurgique. C'est pourquoi ce n'est pas notre pensêe qui opère ces actes... Sans que nous y pensions, en effet, les signes eux-mêmes, par euxmêmes, opèrent leur oeuvre propre, et 1 'ineffable puissance des dieux, que ces signes concernent, reconnaît ses propres copies elle-même par elle-même, sans avoir besoin d'être éveillée par 1'activité de notre pensée. En effet, il n'est pas dans 1'ordre que le contenant soit mis en branle par le contenu, le parfait par l'imparfait, le 
tout par les parties. Nos pensêes ne provoquent donc pas, en les prévenant, les causes divines à s'exercer ; mais elles doivent, avec toutes les dispositions excellentes de l'âme et avec notre pureté, prëexister comme causes auxiliaires ; ce qui éveille proprement le vouloir divin, ce sont les signes divins eux-mêmes ; et ainsi le divin est déterminè par le divin et ne reçoit d'aucun des êtres inférieurs un principe quelconque de son action propre." (Ibid., II, II, 96-98)

"Même I'âme parfaite est imparfaite en regard de l'activité divine... ce sont les dieux seuls qui accordent le succès dans les oeuvres divines." (Ibid., III, 20, 149)

Nous voyons que c'est Dieu lui-même qui introduit leur efficacité dans les éléments du sacrifice. Pour y introduire cette efficacité, il doit rêaliser lui-même le prototype du sacrifice, c'est-à-dire se livrer lui-même à $l$ 'avance dans ses symboles. Tous les autres sacrifices ne seront jamais que la répétition plus ou moins complète et fidèle de cette révélation en acte qui prendra rang soit dans 1 'histoire (par inspiration d'un être humain privilégiê), soit dans le mythe.

d) Ce n'est pourtant pas par caprice que les dieux accordent les bienfaits qui résultent du sacrifice. Ils ne sauraient se retenir d'exercer comme il se doit leur providence. Il y a nécessité pour eux de gouverner avec justice et de communiquer étroitement leur nature à tout ce qui les signifie conformément à leur volonté.

Le sacrifice provoque ici-bas une manifestation des dieux propre à éveiller en l'homne une nature divine semblable à la leur qui demeurait assoupie, ou submergée par les agitations mondaines, au fond de lui-même. Sans cette similitude préalable, rien ne se passe; d'où l'idée que chacun est voué à l'avance à tel ou tel dieu. Celui voué à Asclépios maniera avec succès les incantations curatives, celui voué à Apollon décryptera avec succès les versets prophétiques etc...

"Mieux vaut donc chercher la cause (des sacrifices) dans une amitié, une parenté, une relation qui lie les ouvriers à leurs ouvrages et les générateurs à ceux qu'ils engendrent. Quand donc, sous l'égide de ce principe, nous voyons un animal ou une plante terrestre conserver intacte et pure l'intention de son auteur, alors par cet intermédiaire nous mettons en mouvement, d'une manière appropriée, la cause démiurgique qui, sans rien perdre de sa pureté, domine cette créature. Nombreuses sont ces causes ; les unes sont dans une dépendance immédiate comme les démons; les autres, supérieures, se situent au-dessus, celles des dieux; enfin à leur tête, se trouve la cause unique, la plus vénérable; le sacrifice parfait les met toutes en branle, mais chacune selon le rang qu'elle a reçu, s'y introduit familièrement. $\mathrm{Si}$ au contraire le sacrifice est imparfait, il progresse jusqu'à un certain point, mais est incapable de poursuivre plus avant. 
"Par suite beaucoup croient que les sacrifices sont offerts aux bons dếmons ; beaucoup, aux dernières puissances des dieux ; d'autres aux puissances cosmirues ou terrestres des démons ou des dieux; pour une partie des sacrifices, ils n'ont pas tort, mais il leur échappe que l'ensemble de la puissance et tous les biens remontent finalement à tout le divin." (Jamblique, Ibid., V, 9, 209-210)

e) Certes, comme nous venons de le voir, le sacrifice se répercute du haut en bas de l'échelle des êtres et remonte même jusqu'au Dieu suprême.

Il se situe dans un ensemble d'entités toutes coordonnées les unes aux autres, c'est-à-dire toutes en relation plus ou moins proches les unes avec les autres, unies par les liens d'une sympathie universelle, à l'intérieur duquel rien ne se passe localement qui ne se répercute proportionnellement dans tout le reste.

Cependant, toute interprétation mécaniciste du sacrifice en termes d'échange ou de circulation quasi-physique d'énergies subtiles (le prêtre interviendrait ici pour obtenir là-bas tel effet ; il y aurait au cours du sacrifice telle circulation de "force vitale") est une interprêtation largement incomplète si ce n'est totalement fausse. L'ensemble oũ apparaît le sacrifice n'est pas un ensemble clos d'effets se transformant les uns dans les autres et se répercutant les uns sur les autres dans le déterminisme le plus absolu. C'est un ensemble également peuplé de causes sous forme d'entités souveraines responsables par définition d'intervenir, comme un pilote réorientant la marche de son navire, dans l'évolution du monde.

"Si nous disons que dans un seul vivant, le Tout - qui a partout une seule et même vie - la communauté des puissances semblables, la lutte des contraires, une certaine aptitude de l'agent au patient meuvent ensemble le semblable et 1 'apte, en se répandant de la même façon selon une sympathie même dans les plus êloignés comme s'ils étaient le plus près, on dit de cette manière quelque chose de ce qui se passe réellement et accompagne nécessairement les sacrifices, mais ce n'est pas le véritable mode de sacrifices que 1 'on montre. Car ce n'est pas dans la nature ni dans la nécessité physique que réside 1 'essence divine, au point d'être éveillée par des passions physiques ou par les puissances qui s'étendent à travers toute la nature, mais elle est limitêe en elle-même, à l'écart des ces passions, sans rien de commun avec celles-ci..."

"D'après cela (sacrifice attribué à la vertu des ses éléments naturels), ni la causalité divine ne s'avère-surnaturelle, ni elle ne s'ébranle comme telle à l'occasion des sacrifices; mais comme une cause physique retenue par la matière et attachée physiquement aux corps s'éveille et s'assoupit avec eux, ainsi font ces éléments de la nature. Et si quelque chose de tel dans les essences accompagne (1e 
sacrifice), c'est comme auxiliaire ou condition nécessaire qu'il est attaché aux causes principales." (Ibid., $\nabla, 7$ et $8,207,209$ )

Ainsi donc le sacrifice prêpare au dieu un réceptacle digne de Iui, mais ce qui descend du dieu sur ce rêceptacle (autel, être humain ou espace sacré) est de nature infiniment supérieure à celle du réceptacle. Quelle que soit la répercussion naturelle de ce qui est humainement opéré sur le rêceptacle, quel que soit ce qui est ainsi naturellement amorcé, l'effusion divine qui se produit alors le surpasse considérablement en bienfaits. Dieu rend toujours ainsi "au centuple" ce qui a été envoyé vers lui.

f) Bien que le don des grâces divines apparaisse sans aucune mesure, tant avec les efforts fournis par les hommes pour en bênêficier qu'avec les composantes matérielles du sacrifice, ce serait folie que de les négliger puisque ce sont là, non pas certes les causes fondamentales, mais cependant les conditions nécessaires de 1a manifestation divine.

D'où l'importance accordée à la mise en condition de l'officiant (intégrité et pureté physique, pratique de la vertu, respect de nombreux interdits), à la préparation des lieux, à la stricte observance du rituel et, en particulier, à la fabrication des symboles sacrés et des autels.

En aucun cas, I'imperfection de toute puissance naturelle par rapport à la puissance divine ne conduit les philosophes néoplatoniciens à nêgliger la matière et le corps, encore moins à les mépriser. Ils ne cesseront, au contraire, d'insister lourdement sur les mesures matérielles à prendre pour réussir un sacrifice, puisque c'est là et jusque là que Dieu lui-même'a déposé les signes auxquels il a voulu et continue éternellement de vouloir répondre. Plus éloignées de Dieu sont les réalités considérées, plus puissante est, parallèlement, 1 'action divine déployée pour $s^{\prime} y$ rendre néanmoins présente. Parce qu'elle est la plus éloignée du sommet des êtres, la matière est la plus haute preuve de la grandeur divine dont elle nous donne toute la mesure ; c'est donc au niveau de la matière que nous saurons appeler les énergies salvatrices les plus fortes, celles dont les individus les moins spiritualisés que nous sommes ont précisément besoin pour accéder à la Lumière intelligible.

"L'abondance de force des êtres les plus élevés leur donne chaque 
Le sacrifice du point de vue néoplatonicien

fois sur l'ensemble cette supérioritê d'être présents à tous également de la même manière sans obstacle ; par suite, selon ce principe, les premiers resplendissent dans les derniers et les immatériels sont immatériellement présents aux matériels. Que l'on ne s'étonne donc pas si nous disons pure et divine une certaine matière ; issue, en effet, elle aussi du père et Démiurge de l'Univers, sa perfection la rend apte à recevoir les dieux. Et, en même temps, rien n'empêche les êtres supérieurs de pouvoir illuminer leurs inférieurs, rien non plus, par conséquent, n'écarte la matière de la participation aux biens les plus élevés, de sorte que si elle est parfaitement pure et boniforme, elle n'est pas impropre à recevoir les dieux ; comme il fallait, en effet, que le terrestre lui-même ne fût pas privé de la participation divine, la terre aussi a reçu de celle-ci un lot divin, capable d'accueillir les dieux.

"Ce que voyant, la théurgie, en découvrant ainsi d'une manière générale selon l'appropriation les réceptacles adaptés à chacun des dieux, aime à tresser ensemble pierres, herbes, animaux, aromates, d'autres objets sacrés parfaits et déiformes, pour faire ensuite de tout cela un réceptacle intégral et pur.

"Car il ne faut pas repousser toute matière, mais seulement celle qui répugné aux dieux ; on choisira, au contraire, celle qui leur est apparentée comme capable de convenir à l'édification de leurs demeures, à la consécration des statues et aussi aux rites des sacrifices. Autrement, en effet, les endroits de la terre, ou les homes qui viennent y habiter, ne sauraient avoir part à la réception des êtres supérieurs, si un tel fondement n'avait d'abord été posé ; et il faut en croire les discours secrets : grâce aux contemplations bienheureuses, une certaine matière est livrée par les dieux, qui est, je suppose, connaturelle à ceux-mêmes qui la donnent; ainsi le sacrifice d'une matière de cette espèce éveille les dieux pour qu'ils apparaissent et les invite aussitôt à se laisser saisir ; il les accueille quand ils se présentent et les montre parfaitement." (Jamblique, Tbid., v, $23 / 232-234)$

L'un des traités hermétiques, l'Asclépius, appartenant au même courant théurgique d'inspiration égyptienne et moyen-orientale, contribueà êclairer notre propos. Nous y lisons (sections 37 et 38) :

"Nos premiers ancêtres (égyptiens)... inventèrent l'art de faire des dieux ; puis, l'ayant trouvé, ils y attachèrent une vertu appropriée, qu'ils tiraient de la nature matérielle ; et en mêlant cette vertu à la substance des statues, comme ils ne pouvaient créer proprement des âmes, après avoir évoqué des âmes de démons ou d'anges, ils les introduisirent dans leurs idoles par des rites saints et divins, en sorte que ces idoles eussent le pouvoir de faire du bien et du mal."

"... Car les dieux terrestres et matériels entrent facilement en colère, puisque les hommes les ont fabriqués et composés de l'une et 1 'autre nature"...

"Et de ces dieux qu'on nomme terrestres, O Trismégiste, de quelle sorte est la propriété ?

"Elle résulte, Asclépius, d'une composition d'herbes, de pierres et d'aromates qui contiennent en eux-mêmes une vertu occulte d'efficacité divine. Et si l'on cherche à les réjouir par de nombreux sacrifices 
des hymnes, des chants de louanges, des concerts de sons très doux qui rappelent 1 'harmonie du ciel, c'est pour que cet élément céleste qui a été introduit dans l'idole par la pratique répétée des rites célestes puisse supporter joyeusement ce long séjour parmi les hommes. Voilà comment 1 'homme fabrique les dieux."

Je puis affirmer, sans risque d'erreur, que de telles paroles ne manqueraient pas d'être approuvêes par les prêtres traditionnels evhé ou fon en ce qui concerne le culte des tro ou des vodu.

Elles nous montrent en quoi, par l'artifice de fabrication des dieux, par la composition des idoles et des autels en correspondance avec certains esprits naturels ou désincarnés, de façon qu'ils soient amenés à y apparaître, le culte africain peut être qualifié de "fétichiste".. J'ai bien peur, en effet, que la récusation actuelle du terme de "fétiche", sous prétexte qu'il rêduirait la religion africaine à des pratiques de sauvages, ne cache une méconnaissance tout aussi profonde de cette religion due à ce que l'on estimerait, a priori, en vertu d'un rationnalisme étriqué, qu'une fabrication des dieux comme celle qui vient d'être décrite est absurde et ne saurait donc leur être attribuée - a fortiori trouver justification dans leur système de pensée - sans leur faire injure.

La philosophie néoplatonicienne nous aide à comprendre que derrière un fétichisme de cette sorte (croyance en l'affinité de certains esprits avec certaines compositions matérielles et accueil de ces esprits par manipulation de telles compositions) se cache une philosophie fort complexe et, qui sait, beaucoup plus subtile, beaucoup plus logique et beaucoup plus objective que la nôtre.

Elles nous invite à ne pas "néantiser" les pratiques fétichistes, à ne pas fermer les yeux devant elles comme s'il s'agissait de pratiques honteuses, mais à entreprendre sérieusement leur analyse. Car c'est 1à que nous voyons mis en oeuvre - et que nous avons donc le plus de chances de mettre à jour - les systèmes classificatoires traditionnels des minéraux, des végétaux et des animaux.

Sous leur aspect matériel, les "fétiches" sont avant tout des signes complexes, codifiant à leur manière - à 1'aide d'élêments reprêsentatifs de telle ou telle classe appartenant à tel ou tel plan un certain aspect de la puissance divine (un certain aspect de la totalité des puissances) qu'ils ont, de ce fait, pouvoir d'attirer ou du moins d'inspirer. Ils nous offrent ainsi le moyen de transcrire sur 
cartes perforées la collection de toutes les puissances divines dont les membres d'une sociétẻ étudiẻe sont enclins à solliciter 1'intervention.

Il est clair que le système où ils s'insèrent est beaucoup plus riche que les vagues notions religieuses à travers lesquelles nous voudrions les comprendre. De ces vagues notions aux conceptions qui les justifient, il y a la même différence qu'entre l'appréhension personnifiante des forces de la nature (analogue à 1 'appréhension personnifiante des forces divines que nous avons) et la science physique (analogue à la science religieuse des prêtres et devins traditionnels).

g) Nul ne saurait passer soudainement de l'état où il se trouve à l'état de développement spirituel le plus élevé. Un tel but n'est atteint que par un cheminement progressif qui, si rapide qu'il soit, et malgré les grâces transcendantes reçues à chaque moment, ne peut être accompli que par étapes.

Ce qui est le plus utile à un individu de niveau donné, ce sont les forces divines émanées de Dieu lui-même ou des êtres qu'il commande jusqu'à ce niveau. II n'a que faire, pour commencer, des forces qui ne sont émanées que jusqu'à des niveaux supérieurs au sien. Jusqu'à ce qu'il se soit purifié de toute attache avec la matière, il conviendra qu'il mette en oeuvre les puissances qui s'exercent sur le plan matériel avant de mettre en oeuvre les puissances qui s'exercent sur des plans de plus en plus subtils. Il faut done prendre contact d'abord avec les démons, les héros, les dieux cosmiques etc... avant de communiquer de plus près avec les dieux de rang supérieur et a fortioni avec le Dieu suprême lui-même.

De ce point de vue, le culte des démons ne nuit nullement au culte de Dieu, il en est l'inévitable préparation. Gardons-nous donc d'opposer une religion s'adressant à des "dieux terrestres" ou fétiches, fabriqués comme nous l'avons vu, à une religion s'adressant au Dieu. unique; ce ne sont là que deux aspects d'un même mouvement de remontée vers Dieu, I'un concernant le grand nombre, l'autre ne concernant qu'un petit nombre.

Nous lisons dans Saint Augustin (De Civitate dei, x, 21, p. 435, Dombart) que "d'après cette opinion qu'il ne donne pas comme sienne mais comme venant d'ailleurs, Porphyre écrit : "Un dieu bon ou un bon "gênie ne vient pas dans un homme si le mauvais n'a d'abord été apaisé." 
"Quand, au contraire, nous sommes enchaînés à ce corps testacê, nous sommes retenus par la matière et corporels; à nouveau donc voici

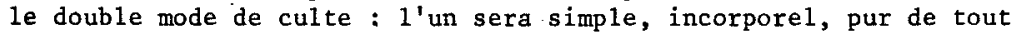
devenir : il échoit aux âmes immaculées ; l'autre, infecté par les corps et toutes les opérations matérielles : il convient aux âmes non purifiées ni affranchies de tout devenir. Pour les sacrifices aussi, par conséquent, je pose deux espèces : celle des homnes entièrement purifiés, qui ne peuvent se réaliser que rarement, comme dit Héraclite, dans le cas d'un seul homme ou d'une élite qui se compterait ; l'autre matérielle, corporelle, constituée par le changement, telle qu'elle s'adapte à ceux que le corps retient encore. Si donc à des cités ou à des peuples non affranchis de la génération et la société contractée avec les corps, on n'accorde un pareil mode de cérémonies, on manquera à la fois les deux sortes de biens, immatériels et matériels, faute de recevoir les uns et d'offrir aux autres ce qui leur est propre. De plus, c'est selon ce qu'il est, non selon ce qu'il n'est pas, que chacun s'occupe du rite; celui-ci ne doit donc pas dépasser la mesure propre du fidèle." (Jamblique, De mystêriis, V, $15 / 219-220$ )

"Ainsi donc, ce qui se produit en un seul homme à grand'peine et tardivement, au sommet de 1 'art hiëratique, il ne faut pas le déclarer commun à tous ni l'attribuer immédiatement à ceux qui abordent la théurgie ou qui sont au milieu de 1a course ; car ceux-1à, d'une manière quelconque, donnent un caractère corporel à leur pratique de la piété." (Ibidl., V, $20 / 228)$

"Eh quoi ! le comble de l'art hiêratique ne se porte-t-il pas vers 1 'Un qui est au sommet de toute la foule (des divinités), ne cêlèbret-il pas à la fois, en lui et avec lui, la multitude des essences et des principes ? Tout à fait, rêpondrais-je; mais cela n'arrive que très tard et à une petite êlite; et il faut s'estimer heureux si l'on en jouit enfin au soir de sa vie." (Ibid., v, 22 / 230-231)

S'il en est ainsi - et du fait que tous les bienfaits descendent de l'Être suprême - le sacrifice mettra en branle, de proche en proche, toute la hiérarchie des êtres supérieurs ; tous se trouveront concernés et tous devront être appelés à y participer.

"Puis donc que, avant que les dieux ne soient là, toutes les puissances qu'ils supposent se mettent préalablement en branle, et quand ils vont se diriger vers la terre, les guident et les précèdent processionnellement, si l'on n'a pas imparti à tous leur dû et salué chacun selon l'honneur qui lui revient, on s'en va non initié et privé de la communication des dieux... Les omissions, si minimes soient-elles, renversent toute 1 'oeuvre du culte, comme dans l'échelle musicale une

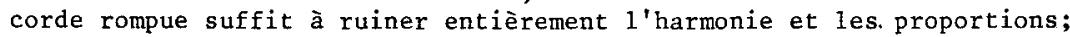
de même donc que, dans les descentes visibles des dieux, manifeste est le tort que se font ceux qui laissent sans honneur quelqu'un des êtres supérieurs, de même aussi, quand ils assistent invisiblement aux sacrifices, il ne faut pas honorer $1^{\prime}$ un sans l'autre, mais bien chacun selon le rang qui lui est échu. Qui en laisse sans sa part d'honneurs bouleverse $1^{\prime}$ ensemble et déchire le dispositif unique et total ; il ne rend donc pas (seulement), comme on pourrait le croire, la réception imparfaite, c'est tout le rite qu'il renverse absolument..."

"Comme un ordre qui unit en un seul ensemble plusieurs rangs, 
Le sacrifice du point de vue néoplatonicien

1 'accomplissement des sacrifices, pour être sans défaut et intégral, doit s'attacher à toute la classe des êtres supérieurs. Mais, comme cette classe est nombreuse, complète et développée sur plusieurs rangs, le rite aussi doit en imiter la variété en faisant appel à toutes les puissances." (Ibid., V, 21-22/ 228-231)

Nous retrouvons là l'une des grandes constantes des cérémonies du culte africain : l'appel, en sacrifiant à telle ou telle divinité (ou fétiche), du Dieu suprême et de toutes les puissances divines qui lui sont subordonnées : ciel et terre, nuit et jour... les quatre orients... les dieux de lignage et les autres dieux des environs, les ancêtres, les génies protecteurs etc..., tous y passent selon leur rang, sans compter 1'appel, par mesure de prudence, de "tous ceux que l'on aurait pu omettre de nommer", le prieur chargeant une divinité messagère de les appeler en son nom. Nous y retrouvons la croyance que tout sacrifice à un dieu ou aux ancêtres remonte jusqu'au Dieu suprême qui est seul 1'ultime cause des bienfaits qui sont finalement accordés.

Sans avoir eu la prétention de vous y introduire, j'espère vous avoir fait sentir à quel point la réflexion néoplatonicienne, surtout celle qui va de Jamblique à Proclus et qui place la théurgie au premier plan - parce qu'elle fut menée en confrontation permanente avec les réalités égyptiennes et proche-orientales, examinées à la lumière du rationnalisme grec - cadre d'une part étroitement avec la mentalité africaine, mais répond aussi à nos exigences scientifiques de clartê logique et de formalisation.

S'il est une école de pensée dont nous devons nous inspirer rejetant au rébut tous ces concepts horriblement flous de "manà", "force vitale", "numineux", "âme" par simple opposition au corps, etc. .. - pour aborder les religions africaines avec toutes les chances d'en saisir et la grandeur et les moindres subtilités, c'est bien celle-là.

Dans l'immédiat, en ce qui concerne l'étude du sacrifice, les néoplatoniciens nous enseignent que nous n'y comprendrons rien tant que nous éviterons d'entrer dans le détail des signes et des symboles qui, dans l'intention des adeptes d'un culte, établissent le contact avec les dieux. Nous pouvons noyer la réalité religieuse sacrificielle sous des flots d'explications de type sociologique, psychanalytique, marxiste, etc... sans jamais l'atteindre autrement que dans ses 
conditions matérielles banales ou dans ses formes dénaturées. Mais, pour en saisir l'essentiel, il nous faudra identifier en l'homme autre chose qu'une conscience flasque ballottée par des idéologies ou qu'un inconscient "poubelle" tourmenté par le refoulement de pulsions instinctuelles, en un mot autre chose qu'un pur effet : un être libre au coeur d'un univers irréductible, prenant figure en profondeur selon ses propres lois. De plus, nous ne pourrons nous dispenser d'une véritable théologie scientifique du genre de celle mise en chantier par Proclus.

Concrètement, nos recherches devront porter sur les systèmes classificatoires des populations étudiées et, pour tout dire, sur le fonctionnement de leur système de pensée, selon le but même de notre laboratoire. Ce sont eux qui nous diront ce que représente le sacrifice et nous expliqueront le pourquoi des rites.

Aussi longtemps que nous n'aurons pas abouti dans ce patient travail de décryptage des symboles que nous poursuivons tous à notre manière, aussi longtemps que nous n'aurons pas assimilé les principales connaissances des grands devins, etc... ce qui nous engage bien plus que du bout des lèvres et du bout de la plume, stimulons-nous mutuellement dans nos recherches, mais ne nous faisons pas trop d'illusion sur la valeur des faits que nous pouvons mettre en discussion.

\section{Réponses aux principales objections.}

J. Rouch : Le caractère abstrait des spéculations néoplatoniciennes donne l'impression d'un jeu de 1 'esprit et fait apparaître une problématique néoplatonicienne qui n'est pas celle des Africains. Aucun Africain traditionnel désireux de transmettre son savoir ne le ferait dans le cadre d'un enseignement philosophique.

A. de Surgy : Aucun pythagoricien, aucun prêtre de Dionysos, aucun initié d'Eleusis ne s'est avisé non plus à divulguer l'enseignement des "mystères". Et je ne connais pas de traité de Jamblique ou de Proclus destiné à apprendre comment évoquer Apollon ou Hécate. Cela n'a pas empêché une réflexion philosophique, nourrie des mystères, de se développer parallèlement aux mystères.

Selon Proclus (In Plat. Théol., I, 4 ; Budé pp. 19-20), il existe quatre modes d'exposition des principes divins, et le mode scientifique et philosophique, qui est plus spécialement celui de Platon, côtoie non seulement un mode symbolique, celui d'Orphée, un mode numérique et mathêmatique, celui de Pythagore, mais encore un mode ésotérique 
qui est celui des mystères.

Quant au "jeu de l'esprit", il vise à apprendre à penser et à se munir de concepts stables dont l'universalité, et donc le caractère scientifique, vient de ce qu'ils ont été dêfinis indêpendamment de 1 'expérience. La spéculation néoplatonicienne est un peu à la science religieuse ce que la mathématique est à la physique.

Voici ce que Platon fait dire à Parménide et à Pythodore au sujet du pur exercice dialectique :

"Entraîne-toi et exerce-toi davantage, pendant que tu es jeune, à ce que la foule juge inutile et qu'elle appelle des bavardages, sinon la vérité t'échappera." (Parménide, $135 \mathrm{~d}$ )

"La foule ne sait pas que, sans cette revue universelle et ce vagabondage, il est impossible de rencontrer la vérité et d'acquérir 1'inte11igence." (Ibid., 136 e)

Et Proclus nous affirme (In Plat. Theol. I, 2 ; Bude, p. 10) :

"Tant que 1 'on ne s'est pas livré à cette espèce de divagation dans les raisonnements, la connaissance intelligible dans les classes divines et de la vérité qui réside en elles est difficile et impraticable."

L. de Heusch et Ph. Jespers : Platon serait un des principaux responsables de la dépréciation de la vie matérielle et du corps qui nous fut ensuite inculquée par le christianisme. Cette dépréciation s'illustre dans le mythe de 1 a caverne. Le but est de délivrer 1 'ame emprisonnée dans le corps, d'éviter d'être "infecté par le corps" (expression de Porphyre). Nous ne trouvons pas en Afrique un tel mépris du plan matêriel.

A. de Surgy : La portêe du mythe de la caverne et la signification de la matière et du corps chez Platon et ses successeurs sont bien mal compris. Comment croire qu'un philosophe qui méprise le corps puisse recommander de :

"... ne jamais mouvoir l'âme sans le corps, ni le corps sans 1 'âme, afin que, se défendant 1 'un contre l'autre, ces deux parties gardent leur équilibre et leur santé. Il faut donc que le mathématicien et quiconque exerce énergiquement quelqu'activité intellectuelle, donne aussi du mouvement à son corps et qu'il pratique la gymastique. .. C'est selon la même règle qu'il faut prendre soin également des différentes parties du corps et de l'âme en imitant la forme de I'univers tout entier" (Timée, 88 b et c).

Quant à Proclus, il écrira:

'La classe des corps qui participent de l'âme intellective, en tant qu'elle est corporelle, est elle aussi, je présume, divine (car la communication de la lumière s'étend à tout l'intervalle depuis le haut jusqu'aux dernier degrés de la participation); toutefois le corps $n$ 'est pas divin de manière absolue..." (In Plat. Theol., I, 14, Budê, p. 67)

"Toutes (les parties du monde), jusqu'à la matière, participent

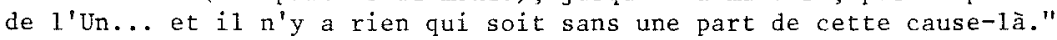
(Ibid., I, 15, Budé, p. 70)

"D'après Platon, la matière procède de l'Un et de l'infinité qui précède l'un-qui-est... C'est pourquoi la matière est bonne sous 
quelque point de vue..." (In Timaeum, I, 385, 10-16)

L. de Heusch : Le dualisme platoninicien et le pessimisme qui 1 'accompagne en ce qui concerne les réalités biologiques s'accorde mal avec la valorisation africaine de la vie en ce monde-ci et non dans l'autre. L'Africain n'est pas attiré par une vie contemplative.

A. de Surgy : On oublie que le dualisme platonicien et néoplatonicien est dominé par un monisme encore plus rigoureux.

H. D. Saffrey, traducteur de Proclus (In Plat. Théol., Budé, p. 131, ncte 2), nous dit que "le critère du véritable platonisme est, selon Proclus... la doctrine de 1 'Un (ou du Bien) comme principe premier".

Proclus dira lui-même :

I)

"Toute pluralité participe à l'Un sous quelque mode." (El Théol.,

"Toute pluralitê est subordonnée à l'Un." (EZ. Théol., 5)

"Il faut bien que le principe premier de tous les êtres soit participé par tous, puisqu'il ne peut jamais être séparé d'aucun d'eux, étant cause de tout ce qui est dit exister de quelque manière." (In Plat. Théol., I, 3, Budé, p. 14)

Toute chose étant liée à 1 'Un, comment supposer une coupure quelconque dans la totalité, y séparant le bien du mal... La matière ellemême procède de l'Un par surabondance infinie du pouvoir de l'Un :

"Le divin, en effet, n'est absent de rien, mais il est présent également à tous. C'est pourquoi, même s'il s'agit d'êtres de dernier rang, on découvrira en eux la présence divine. Car l'Un est partout.." (In Timaeum, I, 209)

"S'il est une divinité purificatrice, il doit y avoir aussi une action purificatrice dans les âmes, les animaux, les plantes et les pierres... La pierre participe à la puissance purificatrice selon le seul mode corporel... Ainsi la série entière tient une puissance identique d'une cause divine unique." (El. Théol., th. 145)

D'autre part, l'Un et le Multiple, qui se trouvent en opposition, ne sont jamais que les deux pôles d'une totalité organique unifiée par un principe qui leur est encore supérieur. Et le modèle biologique est tout aussi néoplatonicien qu'africain. Plotin $\left(2^{\circ}\right.$ Ennéade, livre III) nous dira que :

"L'univers est un animal un et multiple, tout y est coordonné." Puis ( $2^{\circ}$ Ennéade, livre VII) :

"Tout conspire à un but unique... dans 1 'univers il faut un principe unique pour rendre Un cet être multiple, pour en faire l'animal un et universe1... De même que dans le corps humain chaque organe a sa fonction propre, de même dans 1 'univers les êtres ont chacun leur rôle particulier."

Enfin, il est vrai que Plotin qualifie parfois la matière de "mal pur" ( $1{ }^{\circ}$ Ennéade, 8,3 et 39 ). Mais jugeons plutôt le néoplatonisme dans son ensemble et non sur un aspect de son développement dialectique. Se retrancher de la matière et du corps y apparaît plutôt comme un acte de souveraineté sur la matière et sur le corps qui est tout à 1 'opposé d'une coupure. 
Nous trouvons nettement affirmées deux voies spirituelles complémentaires (cf. Proclus, In Plat. Théol., II, 5 et 6) :

- la voie des négations, par laquelle 1 'homme remonte à son principe, et qui correspond à la conversion vers l'Un.

-- la voie de l'analogie par laquelle l'homme se rend semblable à Dieu, c'est-à-dire participe avec lui à l'expression de la puissance divine jusque dans la matière, et qui correspond à la procession des êtres à partir de 1 'Un.

L'homme ne peut, dans un temps, se nourrir de Dieu (Un) que pour, dans un second temps, s'étant assimilê Dieu, se comporter comme Dieu qui est Bien, c'est-a-dire principe qui se donne sans limite, sans jamais rien attendre en retour.

De sorte que 1a thèse "pessimiste" est aussitôt compensée par 1 'antithèse "optimiste", leur lieu de synthèse étant défini comme permanence ou "manence", état d'union ou de coincidence avec 1'Un sans autre nom.

J. Rouch : Je veux bien admettre, avec G. Dieterlen, que le sacrifice dogon, y compris un simple sacrifice de margouillat, n'est jamais que la répétition du sacrifice primordial du Nommo ; je veux bien admettre que, selon cextaines idéologies que je crois importées, 1e sacrifice élève vers dieu, mais il est loin d'en être ainsi pour tous. Ce qui justifie le sacrifice songhay est l'idée qu'il faut céder une vie pour en obtenir une autre et que l'homme bénéficie de la vie en échange de la mort de quelque chose.

Pour les Songhay, le Dieu suprême n'est qu'un trompeur, un usurpateur qui a pris le pouvoir en détournant à son profit le culte antérieur des divinités de la nature. En échange, il promet une vie éternelle qui est la négation même de la vie. Le mystère suprême est la connaissance du nom de la Mère de Dieu. G. Dieterlen a elle-même retrouvé cette Mère sous les traits des quatre éléments.

A. de Surgy : La justification du sacrifice en termes d'échange (de la vie contre une mort) me paraît simpliste. Elle relève de l'imagerie populaire plutôt que de l'enseignement traditionnel. Nous devons aller au-delà de cette imagerie de l'institution sacrificielle qui n'aurait jamais survécu à travers les âges, à tous les niveaux, sans être justifiée et accrochée à d'autres raisons.

En général, ce qui est envoyé vers les dieux est un message, non un don. L'animal sacrifié, ou plutôt l'âme libérée du corps de 1 'animal sacrifié, n'est que le support d'un message. Une mise à mort provoque certains courants; ce sont là des vecteurs de communication avec les êtres cosmiques supérieurs, mais en aucun cas une monnaie d'échange; le don reste orienté de haut en bas, de la cause vers les effets. Nous sommes incapables de donner quoi que ce soit à Dieu ou aux êtres divins si ce n'est d'entrer dans leurs intentions en nous soumettant à eux.

Les Songhay ont le droit d'exprimer à leur manière une critique de leur religion dominante. Mais, du point de vue théologique, un dieu qui a une mère et qui est trompeur n'est pas vraiment le Dieu suprême. C'est un dieu immanent aux affaires du monde, qui participe au devenir ; ce n'est jamais qu'une divinitê triomphante ou, au mieux, le plus grand des dieux cosmiques.

Des distinctions élémentaires s'imposent. Or, précisément, la spéculation néoplatonicienne nous définit le champ universel des possibles et nous invite à y situer les notions religieuses de tel ou tel 
groupe ethnique, ce qui nous en donne un repérage absolu.

Sachons, de préférence, si les caractëristiques du dieu dont nous parlons le rapproche de l'Un, ou de l'Être suprême, ou du Temps Eternel, ou de $1^{\prime}$ Intellect Démiurgique, ou du Démiurge cosmique, ou du principe suprême du monde d'en-bas,... ou du principe inférieur du monde d'en-bas. Car en mélangeant toutes ces notions, nous ne pourrons jamais analyser correctement les faits et converser entre nous.

La Mère de Dieu sous les traits des quatre éléments... qu'est-ce que cela signifie donc ? Bien entendu, cela ne signifie rien, du moins rien de précis qui puisse entrer dans le discours scientifique, pour qui ne sait préciser ce que sont les éléments, ce qu'est Dieu, ce que sont les dieux et ce qu'est une mère de dieu.

Pour comprendre ce que veulent parfois dire nos informateurs, il faut avoir élaboré en nous des structures d'accueil, il faut savoir nous aventurer dans des spéculations thëologiques, ce ẹn quoi les néoplatoniciens peuvent puissamment nous aider.

G. Dieterlen : Toute religion cherche à expliquer le monde sensible en devenir par un acte de création. Or à qui donner la primauté, à l'oeuf ou à la poule ? A une Mère primordiale ou au Dieu qui en est finalement accouché ? Au départ, nous avons un Dieu qui est à 1 a fois sa propre matière; il est sa propre puissance génératrice, le produit de cette puissance et celui qui la féconde. Qui privilégier ? Les puissances de la Nature et la Mère des dieux ? Ou bien les puissances de la Surnature et un Dieu mâle, père ou fils ?

A. de Surgy : Ce problème métaphysique universel n'est évidemment pas laissé sans réponse par la philosophie néoplatonicienne. Cette réponse n'est pas simple, mais contribue à éclairer la pensée religieuse africaine.

I1 existe des causes antécédentes par lesquelles les êtres se donnent mutuellement naissance les uns aux autres et se transforment les uns dans les autres. Ces causes tournent en rond et ne connaissent aucune hiérarchie. Mais il existe aussi des causes retranchées de leurs effets, et notament une cause suprême donnant hiérarchiquement naissance, de proche en proche, à toute la série des êtres. L'ensemble est articulé en un seul et même système où le monde n'a point de commencement dans le temps et où l'acte de création est perpétuel. 